\title{
Correlation Functions for Lattice Integrable Models
}

F. Smirnov

In this lectures I consider the problem of calculating the correlation functions for XXZ spin chain. First, I explain in details the free fermion case. Then I show that for generic coupling constant the fermionic operators acting on the space of quasi-local fields can be introduced. In the basis generated by these fermionic operators the correlation functions are given by determinants as in the free fermion case.

Keywords: Quantum integrable models, correlation functions, exactly solvable models of statistical physics.

\section{Lecture 1.}

These lectures are based on a series of papers written in collaboration with H. Boos, M. Jimbo, T. Miwa, Y. Takeyama $[1,2,3,4,5,6,7,8]$.

Consider the infinite XXZ spin chain with the Hamiltonian

$$
H_{\mathrm{XXZ}}=\frac{1}{2} \sum_{k=-\infty}^{\infty}\left(\sigma_{k}^{1} \sigma_{k+1}^{1}+\sigma_{k}^{2} \sigma_{k+1}^{2}+\Delta\left(\sigma_{k}^{3} \sigma_{k+1}^{3}-1\right)\right) \text {, }
$$

where $\sigma^{a}(a=1,2,3)$ are Pauli matrices and $\Delta=\cos \pi v$.

I shall consider the case $|\Delta| \leq 1$, so, $v$ is real: $0<v<1$. I also use the notation

$$
q=e^{\pi i v}
$$

The ground state of the Hamiltonian will be denoted by $\mid$ vac $\rangle$. I shall later briefly explain how this vector is found, but now let me formulate the main problem.

Introduce

$$
S(k)=\frac{1}{2} \sum_{j=-\infty}^{k} \sigma_{j}^{3}
$$

and let $\alpha$ be a parameter. We consider the normalized vacuum expectation values:

$$
\frac{\left\langle\operatorname{vac}\left|q^{2 \alpha S(0)} \mathcal{O}\right| \mathrm{vac}\right\rangle}{\left\langle\operatorname{vac}\left|q^{2 \alpha S(0)}\right| \mathrm{vac}\right\rangle},
$$

where $\mathcal{O}$ is a local operator. The locality of $\mathcal{O}$ implies that the operator $q^{2 \alpha S(0)} \mathcal{O}$ stabilizes: there exist integers $k, l$ such that for all $j>l$ (resp. $j<k$ ) this operator acts on the $j$-th lattice site as 1 (resp. $\left.q^{\alpha \sigma^{3}}\right)$. If $k$ (resp. $l$ ) is the maximal (resp. minimal) integer with this property, $l-k+1$ will be called the length of the operator $q^{2 \alpha S(0)} \mathcal{O}$ and denoted by lenght $\left(q^{2 \alpha S(0)} \mathcal{O}\right)$.

Let me start with the free fermion case $\Delta=0(q=i)$. In this case we have

$$
H_{X X}=\frac{1}{2} \sum_{k=-\infty}^{\infty}\left(\sigma_{k}^{1} \sigma_{k+1}^{1}+\sigma_{k}^{2} \sigma_{k+1}^{2}\right) .
$$

In this case the model can be easily solved by introducing the fermions:

$$
\psi_{k}^{ \pm}=\sigma_{k}^{ \pm} e^{ \pm \pi i S(k-1)},
$$

which satisfy canonical commutation relations:

$$
\left[\psi_{k}^{\varepsilon_{1}}, \psi_{k}^{\varepsilon_{2}}\right]_{+}=\delta_{k, l} \delta^{\varepsilon_{1}+\varepsilon_{2}, 0} .
$$

The Hamiltonian takes the form:

$$
H_{X X}=i \sum_{k=-\infty}^{\infty}\left(\psi_{k}^{+} \psi_{k+1}^{-}+\psi_{k}^{-} \psi_{k+1}^{+}\right) .
$$

Consider a finite chain of length $N$ with periodical boundary conditions (we assume that $N$ is even):

$$
H_{X X}^{(N)}=i \sum_{k=N / 2}^{N / 2-1}\left(\psi_{k}^{+} \psi_{k+1}^{-}+\psi_{k}^{-} \psi_{k+1}^{+}\right)
$$

$\psi_{N / 2}^{ \pm}=e^{\mp \frac{\pi i}{2} S} \psi_{-N / 2}^{ \pm}, S$ is the total spin. Introduce the Fourier transform:

$$
\psi^{ \pm}(\zeta)=\frac{1}{\sqrt{N}} \sum_{k=N / 2}^{N / 2-1} \psi_{k}^{ \pm}\left(\frac{1+\zeta^{2}}{1-\zeta^{2}}\right)^{ \pm k} .
$$

I apologize for the strange parametrization of the momentum, but it will be useful in the generic case. We have

$$
\left[\psi^{ \pm}\left(\xi_{1}\right), \psi^{ \pm}\left(\xi_{2}\right)\right]_{+}=0,
$$

$$
\begin{aligned}
{\left[\psi^{+}\left(\zeta_{1}\right), \psi^{-}\left(\zeta_{2}\right)\right]_{+}=} & \frac{1}{N}\left(\frac{1-\zeta_{1}^{2}}{1+\zeta_{1}^{2}}\right)^{N / 2}\left(\frac{1+\zeta_{2}^{2}}{1-\zeta_{2}^{2}}\right)^{N / 2} \\
& \cdot \frac{\left(\frac{1+\zeta_{1}^{2}}{1-\zeta_{1}^{2}}\right)^{N}\left(\frac{1-\zeta_{2}^{2}}{1+\zeta_{2}^{2}}\right)^{N}-1}{\left(\frac{1+\zeta_{1}^{2}}{1-\zeta_{1}^{2}}\right)\left(\frac{1-\zeta_{2}^{2}}{1+\zeta_{2}^{2}}\right)-1} .
\end{aligned}
$$

So, introducing $\tau_{j}$ as solutions to

$$
\frac{1-\tau_{j}^{2}}{1+\tau_{j}^{2}}=e^{\frac{2 \pi i}{N} j}, j=-N / 2, \ldots, N / 2-1,
$$

we have:

$$
\left[\psi^{+}\left(\tau_{j}\right), \psi^{-}\left(\tau_{j}\right)\right]_{+}=\delta_{i, j} .
$$

The Hamiltonian is easily expressed in terms of the Fourier transform:

$$
H_{X X}^{(N)}=\sum_{k=N / 2}^{N / 2-1} \psi^{+}\left(\tau_{j}\right) \psi^{-}\left(\tau_{j}\right) \sin \left(\frac{2 \pi}{N} j\right) .
$$


As usual in fermionic models, there are negative energies which are taken care of by the Dirac trick: rewrite the Hamiltonian as

$$
\begin{aligned}
H_{X X}^{(N)} & =\sum_{k=0}^{N / 2-1} \psi^{+}\left(\tau_{j}\right) \psi^{-}\left(\tau_{j}\right) \sin \left(\frac{2 \pi}{N} j\right) \\
& -\sum_{k=-N / 2}^{-1} \psi^{-}\left(\tau_{j}\right) \psi^{+}\left(\tau_{j}\right) \sin \left(\frac{2 \pi}{N} j\right)+\text { Const }
\end{aligned}
$$

which means that the vacuum must satisfy

$$
\begin{aligned}
& \psi^{+}\left(\tau_{j}\right)|\mathrm{vac}\rangle=0, j=-N / 2, \ldots,-1 \\
& \psi^{-}\left(\tau_{j}\right)|\mathrm{vac}\rangle=0, j=0, \ldots, N / 2-1 .
\end{aligned}
$$

So, if we start with a ferromagnetic vacuum $|0\rangle$ in which all spins are down, the real vacuum $|\mathrm{vac}\rangle$ is obtained by filling the Dirac sea:

$$
|\mathrm{vac}\rangle=\prod_{j=-N / 2}^{-1} \psi^{+}\left(\tau_{j}\right)|0\rangle .
$$

I have considered this simple example in order to explain that even in the free fermion case the vacuum is a rather complicated state if we present it in terms of original spin variables.

Now we have to take the limit $N \rightarrow \infty$. For the Fourier transform we have

$$
\psi_{k}^{ \pm}=\oint_{\zeta^{2}=1}\left(\frac{1-\xi^{2}}{1+\xi^{2}}\right) \psi^{ \pm}(\zeta) \frac{2 d \xi^{2}}{1-\xi^{4}},
$$

and the vacuum satisfies:

$$
\begin{aligned}
& \left.\psi^{+}(\zeta) \mid \text { vac }\right\rangle=0, \text { phase }\left(\frac{1-\xi^{2}}{1+\xi^{2}}\right)<0, \\
& \left.\psi^{-}(\zeta) \mid \text { vac }\right\rangle=0, \text { phase }\left(\frac{1-\xi^{2}}{1+\xi^{2}}\right)<0 .
\end{aligned}
$$

Let me give some explanation about the case of generic $q$. It is well known nowadays that the integrability of the XXZ model is due to its relation to the trigonometric $R$-matrix. The $R$-matrix belongs to the tensor product of the two algebras of the 2 matrices: $R(\zeta) \in \operatorname{Mat}(2, \mathbb{C}) \otimes \operatorname{Mat}(2, \mathbb{C})$ :

$$
\begin{aligned}
R(\xi) & =\frac{1}{\zeta q-\zeta^{-1} q^{-1}} \\
& \left(\begin{array}{cccc}
\zeta q-\xi^{-1} q^{-1} & 0 & 0 & 0 \\
0 & \zeta-\zeta^{-1} & q-q^{-1} & 0 \\
0 & q-q^{-1} & \zeta-\xi^{-1} & 0 \\
0 & 0 & 0 & \zeta q-\zeta^{-1} q-1
\end{array}\right)
\end{aligned}
$$

The $R$-matrix satisfies the Yang-Baxter equation:

$R_{12}\left(\frac{\zeta_{1}}{\zeta_{2}}\right) R_{13}\left(\frac{\zeta_{1}}{\zeta_{3}}\right) R_{23}\left(\frac{\zeta_{2}}{\xi_{3}}\right)=R_{23}\left(\frac{\zeta_{2}}{\xi_{3}}\right) R_{13}\left(\frac{\zeta_{1}}{\zeta_{3}}\right) R_{12}\left(\frac{\zeta_{1}}{\xi_{2}}\right)$,

where as usual I shall denote by $R_{i, j}(\xi)$ the $R$-matrix acting in the tensor product of two copies of $\operatorname{Mat}(2, \mathbb{C})$. I shall also use the notion of an $L$-operator. In this particular case there is no difference between these two things, and I shall explain later what I mean by an $L$-operator in general. Here I consider two copies of $\operatorname{Mat}(2, \mathbb{C})$ one of which is called auxiliary (carrying the index $a$ ), and the other is called quantum (carrying the in$\operatorname{dex} j$ ). So, $L_{a, j}(\xi)$ is $R(\xi)$ as an element of the tensor product of these two algebras.

The most important object of the theory of integrable models is the transfer-matrix:

$$
t_{N}(\zeta)=\operatorname{tr}_{a}\left(L_{a, N / 2-1}(\zeta) \cdots L_{a,-N / 2}(\zeta)\right)
$$

The Yang-Baxter equation implies commutativity:

$$
\left[t_{N}\left(\zeta_{1}\right), t_{N}\left(\xi_{2}\right)\right]=0 .
$$

It is well known that the Hamiltonian of a periodic XXZ chain on $N$ sites is contained in this one-parametric family of operators, but since I shall need some knowledge about higher local integrals of motion let me repeat the derivation of this fact.

It is convenient to introduce

$$
\widetilde{L}_{a, j}(\zeta)=\xi^{-\frac{\sigma_{j}^{3}}{2}} \widetilde{L}_{a, j}(\zeta) \xi^{\frac{\sigma_{j}^{3}}{2}}
$$

Note that we still have

$$
t_{N}(\zeta)=\operatorname{tr}_{a}\left(\widetilde{L}_{a, N / 2-1}(\zeta) \cdots \widetilde{L}_{a,-N / 2}(\zeta)\right)
$$

because $t_{N}(\xi)$ commutes with the total spin. Introduce further

$$
L_{a, j}^{\prime}(\zeta)=\widetilde{L}_{a, j}(\xi) P_{a, j}=1+\frac{\xi^{2}-1}{\xi^{2} q-q^{-1}} h_{a, j},
$$

where

$$
\begin{aligned}
h_{a, j}= & \left(\sigma_{a}^{+} \sigma_{j}^{-}+\sigma_{a}^{-} \sigma_{j}^{+}+\frac{q+q^{-1}}{4}\left(\sigma_{a}^{3} \sigma_{j}^{3}-1\right)\right. \\
& \left.+\frac{q-q^{-1}}{4}\left(\sigma_{a}^{3}-\sigma_{j}^{3}\right)\right) .
\end{aligned}
$$

Note that the operator $h_{a, j}$ (the density of the Hamiltonian) is a projector, so it is easy to see that

$$
L_{a, j}^{\prime}(\xi)=\exp \left(\frac{1}{q+q^{-1}} \log \left(\frac{q-\xi^{2} q^{-1}}{\xi^{2} q-q^{-1}}\right) h_{a, j}\right)
$$

Now we calculate

$$
\begin{aligned}
t_{N}(\xi)= & \vec{L}_{-N / 2, N / 2-1}^{\prime}(\zeta) L_{N / 2-1, N / 2-2}^{\prime}(\zeta) \cdots L_{-N / 2+1,-N / 2}^{\prime}(\zeta) \\
& \times P_{-N / 2, N / 2-1} \cdots P_{-N / 2, N / 2+1}=e^{\sum_{p=1} I_{p}} U
\end{aligned}
$$

where $\vec{L}_{-N / 2, N / 2-1}^{\prime}$ means that the operators from the $-N / 2$ $(N / 2-1)$ tensor component are put to the right (left) of the product of the $L$-operators. It is easy to see that $I_{p}=H_{\mathrm{XXZ}}^{(N)}$, which is the Hamiltonian of the periodic XXZ chain. Moreover, from the Campbell-Hausdorf formula one concludes that

$$
I_{p}=\sum_{j} d_{p, j}
$$


where $d_{p, j}$ acts nontrivially from the $j$-th to the $(j+p)$-th site. These operators are called local integrals of motion: they commute with the Hamiltonian, and they are composed of local densities.

Finally, I would like to say what the ground state of the Hamiltonian of the periodic chain looks like. Denote

$$
L_{a, N / 2-1}(\zeta) \cdots L_{a,-N / 2}(\xi)=\left(\begin{array}{ll}
A_{N}(\xi) & B_{N}(\zeta) \\
C_{N}(\zeta) & D_{N}(\zeta)
\end{array}\right)_{a}
$$

The eigenvectors of the transfer matrix with total spin 0 (a vacuum is among them) are constructed as

$$
C_{N}\left(\tau_{1}\right) \cdots C_{N}\left(\tau_{N / 2}\right)|0\rangle
$$

where $\tau_{j}$ solve the $\mathrm{B}$ the equations:

$$
\left(\frac{1-\xi_{j}^{2}}{1-q^{2} \xi_{j}^{2}}\right)^{N}=\prod_{k \neq j} \frac{\xi_{j}^{2}-q^{2} \xi_{k}^{2}}{\xi_{k}^{2}-q^{2} \xi_{j}^{2}}, \quad j=1, \ldots, N / 2
$$

A vacuum corresponds to a particular solution to these equations which is a continuous deformation from the free fermion case. So, we see that the vacuum is a complicated state which hardly allows a mathematically satisfactory description in the case $N \rightarrow \infty$.

\section{Lecture 2.}

Let us return to our main problem. We want to calculate

$$
\frac{\left\langle\operatorname{vac}\left|q^{2 \alpha S(0)} \mathcal{O}\right| \text { vac }\right\rangle}{\left\langle\operatorname{vac}\left|q^{2 \alpha S(0)}\right| \text { vac }\right\rangle}
$$

Consider the free fermion case. One easily calculates:

$S(0)=-\frac{1}{\pi^{2}} \oint_{\xi_{1}^{2}=1} \oint_{\xi_{2}^{2}=1} \frac{\mathrm{d} \xi_{1}^{2}}{1+\xi_{1}^{2}} \frac{\mathrm{d} \xi_{2}^{2}}{1-\xi_{2}^{2}}: \psi^{+}\left(\xi_{1}\right) \psi^{-}\left(\xi_{2}\right): \frac{1}{\xi_{1}^{2}-\xi_{2}^{2}+0}$,

where : : stands for normal ordering with respect to the creation-annihilation parts of fermions discussed before. For further computation I need the formula:

$$
e^{2 \alpha S(0)}=: e^{Z_{\alpha}}:
$$

This is a simple exercise on normal reordering of an exponent of quadratic form. One finds:

$Z_{\alpha}=$

$$
-\frac{4 \sin \frac{\pi \alpha}{2}}{\pi^{3}} \oint_{\zeta_{1}^{2}=1} \oint_{\zeta_{2}^{2}=1} \frac{\mathrm{d} \xi_{1}^{2}}{1+\xi_{1}^{2}} \frac{\mathrm{d} \xi_{2}^{2}}{1-\zeta_{2}^{2}}: \psi^{+}\left(\zeta_{1}\right) \psi^{-}\left(\xi_{2}\right): \frac{\xi_{1}^{\alpha} \xi_{2}^{-\alpha}}{\xi_{1}^{2}-\xi_{2}^{2}+0},
$$

from this formula one easily derives:

$$
\begin{aligned}
& \frac{\left\langle\operatorname{vac}\left|q^{2 \alpha S(0)} \psi^{+}\left(\zeta_{1}^{+}\right) \cdots \psi^{+}\left(\zeta_{k}^{+}\right) \psi^{+}\left(\zeta_{1}^{-}\right) \cdots \psi^{+}\left(\zeta_{k}^{-}\right)\right| \operatorname{vac}\right\rangle}{\left\langle\operatorname{vac}\left|q^{2 \alpha S(0)}\right| \operatorname{vac}\right\rangle} \\
& =\operatorname{det} \sigma\left(\xi_{i}^{+}, \zeta_{j}^{-}\right)
\end{aligned}
$$

where $\sigma\left(\zeta_{1}, \zeta_{2}\right)$ is the two-point Green function:

$\sigma\left(\zeta_{1}, \zeta_{2}\right)=-\frac{y^{2}}{1-y^{2}} \frac{\left(1+\zeta_{1}^{2}\right)\left(1+\zeta_{2}^{2}\right)}{2\left(\zeta_{1}^{2}+\zeta_{2}^{2}\right)}+\frac{2 y}{1-y^{2}} \frac{\zeta_{1}^{\alpha} \zeta_{2}^{-\alpha}}{\zeta_{1}^{2}+\zeta_{2}^{2}}$, where

$$
y=e^{\frac{\pi i \alpha}{2}} .
$$

I want to rewrite this answer differently, but let me first explain my logic. I appeal to an analogy with Conformal Field Theory (CFT). It is well-known that on long distances the XXZ model is described by CFT with $c=1$. Moreover, the XXZ model can be considered as a perturbation of this CFT by an infinite number of irrelevant operators. Here, contrary to the perturbation of relativistic quantum field theory the flow of the renormalization group goes from infrared to finite distances. The main object for CFT is not the space of states, but the space operators. Following this analogy let me denote by $W_{\alpha, 0}$ the space of the spinless operators for the XXZ model of the form $q^{2 \alpha S(0)} \mathcal{O}$. In the conformal limit this space goes to the space of descendants of the primary field

$$
\varphi_{\alpha}=e^{\frac{i \alpha\left(\beta-\beta^{-1}\right)}{2}(\phi+\bar{\phi})}
$$

here $(\phi+\bar{\phi})$, are two chiral bosonic fields. The descendants are created by the action of two chiral Virasoro algebras:

$$
\begin{aligned}
& {\left[L_{m}, L_{n}\right]=(m-n) L_{m+n}+\frac{1}{12} n\left(n^{2}-1\right) \delta_{m,-n},} \\
& {\left[\bar{L}_{m}, \bar{L}_{n}\right]=(m-n) \bar{L}_{m+n}+\frac{1}{12} n\left(n^{2}-1\right) \delta_{m,-n} .}
\end{aligned}
$$

We have:

$$
\begin{aligned}
& L_{m}\left(\varphi_{\alpha}\right)=\bar{L}_{m}\left(\varphi_{\alpha}\right)=0, \quad m>0, \\
& L_{0}\left(\varphi_{\alpha}\right)=\bar{L}_{0}\left(\varphi_{\alpha}\right)=\Delta_{\alpha} \varphi_{\alpha},
\end{aligned}
$$

where $\Delta_{\alpha}=\left(\alpha\left(\beta-\beta^{-1}\right)\right)^{2} / 8 \pi$ is the anomalous dimension. The space of spinless descendants is spun by the vectors

$$
\bar{L}_{-k_{1}} \cdots \bar{L}_{-k_{p}} L_{-l_{1}} \cdots L_{-l_{p}}\left(\varphi_{\alpha}\right) \text {. }
$$

After perturbation, the descendants acquire the vacuum expectation values. The question which A. Zamolodchikov asked me long ago is whether it is possible to write find a covector $\langle 0| F\left(L_{1}, L_{2}, \ldots, \bar{L}_{1}, \bar{L}_{2}, \ldots\right)$ (here $\langle 0|$ has nothing to do with what was previously used, it is the Virasoro left vacuum $\langle 0| L_{-k}=\langle 0| \bar{L}_{-k}$ for $\left.\geq 0\right)$ such that the vacuum expectation values are given by the scalar products of this covector with descendants. At that time I was unable to answer this question, but in these lectures I shall explain a construction which is very much in the spirit of this point of view.

So, our goal is to start working in the space of the operators and to introduce the operators acting in this space. Let me give some formal definitions. I have already defined the space $W_{\alpha, 0}$. Similarly I define the spaces $W_{\alpha, s}$ of operators $q^{2 \alpha S(0)} \mathcal{O}$ of $\operatorname{spin} s$, and

$$
\mathcal{W}_{\alpha}=\bigoplus_{s=-\infty}^{\infty} \mathcal{W}_{\alpha, s}
$$

Also, we shall shift $\alpha$ by integers, so I introduce the space: 


$$
\mathcal{W}_{[\alpha]}=\bigoplus_{k=-\infty}^{\infty} \mathcal{W}_{\alpha+k}
$$

We shall need an analogue of the left Virasoro vacuum, i.e. a linear functional on $W_{\alpha}$. First, introduce also the linear functional on $\operatorname{End}\left(\mathbb{C}^{2}\right)$ :

$$
\operatorname{tr}^{\alpha}(x)=\frac{1}{q^{\frac{\alpha}{2}}+q^{-\frac{\alpha}{2}}} \operatorname{tr}\left(q^{-\frac{1}{2} \alpha \sigma^{3}} x\right)
$$

with the obvious properties:

$$
\operatorname{tr}^{\alpha}(1)=\operatorname{tr}^{\alpha}\left(q^{\alpha \sigma^{3}}\right)=1 \text {. }
$$

This gives rise to a linear functional on $\mathcal{W}_{\alpha}$

$$
\operatorname{tr}^{\alpha}(X)=\cdots \operatorname{tr}_{1}^{\alpha} \operatorname{tr}_{2}^{\alpha} \operatorname{tr}_{3}^{\alpha} \cdots(X)
$$

Obviously, this functional is well-defined, since any operator from $W_{\alpha}$ stabilizes at $+\infty(-\infty)$ as $I\left(e^{\alpha \sigma^{3}}\right)$.

Introduce the operators

$$
\begin{aligned}
& \Psi_{k}^{ \pm}(X)=-\psi_{k}^{ \pm} X-(-1)^{F(X)} X \psi_{k}^{ \pm} \\
& \Phi_{k}^{ \pm}(X)=\frac{1}{1-y^{\mp 2}}\left(\psi_{k}^{ \pm} X-y^{\mp 2}(-1)^{F(X)} X \psi_{k}^{ \pm}\right),
\end{aligned}
$$

where $F(X)$ is the fermionic number of operator $X$. These operators annihilate respectively the right or left tail of elements of $W_{\alpha}$. They satisfy the canonical commutation relations:

$$
\begin{aligned}
& {\left[\Psi_{k}^{\varepsilon}, \Psi_{l}^{\varepsilon^{\prime}}\right]_{+}=\left[\Phi_{\alpha, k}^{\varepsilon}, \Phi_{\alpha, l}^{\varepsilon^{\prime}}\right]_{+}=0} \\
& {\left[\Psi_{k}^{\varepsilon}, \Phi_{\alpha, l}^{\varepsilon^{\prime}}\right]_{+}=\delta_{\varepsilon+\varepsilon^{\prime}, 0} \delta_{k, j} .}
\end{aligned}
$$

Introduce the Fourier transform:

$$
\begin{aligned}
& \Psi^{ \pm}(\xi)=\sum_{j=-\infty}^{\infty} \Psi_{j}^{ \pm}\left(\frac{1+\xi^{2}}{1-\xi^{2}}\right)^{j}, \\
& \Phi^{ \pm}(\zeta)=\sum_{j=-\infty}^{\infty} \Phi_{j}^{ \pm}\left(\frac{1-\xi^{2}}{1+\xi^{2}}\right)^{j} .
\end{aligned}
$$

We want to use $\Psi_{k}^{ \pm}\left(\Phi_{k}^{ \pm}\right)$for $k>0$ as annihilation (creation). Obviously,

$$
\Psi_{k}^{ \pm}\left(q^{2 \alpha S(0)}\right)=0, \quad k>0 .
$$

There is a problem with the creation operators and the functional $\operatorname{tr}^{\alpha}: \operatorname{tr}^{\alpha}\left(\Phi_{k}^{ \pm}(X)\right)$ does not always vanish. However, one easily derives that

$$
\operatorname{tr}^{\alpha}\left(\Phi_{k}^{ \pm}(X)\right)= \pm \frac{y}{y^{2}-1} \operatorname{tr}^{\alpha}\left(\Psi_{k}^{ \pm}(X)\right)
$$

which allows us to perform all necessary calculations. Without going into further details I rewrite the formula for vacuum expecation values in a new form:

$$
\frac{\left\langle\operatorname{vac} \mid y^{2 S(0)} \mathcal{O}_{>} \operatorname{vac}\right\rangle}{\left\langle\operatorname{vac}\left|y^{2 S(0)}\right| \operatorname{vac}\right\rangle}=\operatorname{tr}^{\alpha}\left(e^{\Omega^{\prime}}\left(\mathcal{O}_{>}\right)\right)
$$

$\Omega^{\prime}=\frac{i}{\sin \frac{\pi \alpha}{2}} \oint_{\zeta_{1}^{2}=1} \oint_{\zeta_{2}^{2}=1}\left(\frac{\zeta_{1}^{\alpha} \xi_{2}^{-\alpha}-1}{\xi_{1}^{2}+\zeta_{2}^{2}} \psi^{-}\left(\xi_{2}\right) \psi^{+}\left(\xi_{1}\right) \frac{\mathrm{d} \zeta_{1}^{2}}{1+\zeta_{1}^{2}} \frac{\mathrm{d} \xi_{2}^{2}}{1-\zeta_{2}^{2}}\right)$

where $\mathcal{O}_{>}$denote local operators living on a positive axis only.

Still I am not quite happy with this formula, because there is a problem with translational invariance. I would like to avoid using $\mathcal{O}_{>}$. I introduce the following operators:

$\left.\mathbf{b}(\zeta)\right|_{W_{a, s}}=$

$$
\begin{gathered}
\frac{2 i^{-s}}{1-(-1)^{s} y^{2}} \zeta^{-\alpha+s} \operatorname{sing}_{\zeta^{2}=1}\left[\Psi^{-}(\zeta) E^{-}(\zeta, \alpha+s) \frac{1}{1+\xi^{2}}\right], \\
\left.\mathbf{c}(\zeta)\right|_{W_{a, s}}=2 y \zeta^{\alpha-s} \operatorname{sing}_{\zeta^{2}=1}\left[\Psi^{+}(\zeta) E^{+}(\zeta, \alpha+s) \frac{1}{1+\xi^{2}}\right],
\end{gathered}
$$

where

$E^{ \pm}(\zeta, \alpha)=$

$$
\exp \left(N\left[\Phi_{\alpha}^{ \pm} \log \left(I-\zeta^{2} M\right) \Psi^{\mp}-\Phi_{\alpha}^{\mp} \log \left(I+\zeta^{2} M\right) \Psi^{ \pm}\right]\right) .
$$

In the last formula we consider $\Phi_{\alpha, j}^{ \pm}\left(\right.$resp. $\left.\Psi_{j}^{ \pm}\right)$as components of a row (resp. column) vector,

$$
M=(1+u)(1-u)^{-1}, \quad\left(u \Psi^{ \pm}\right)_{j}=\Psi_{j+1}^{ \pm},
$$

and $\log \left(I-\zeta^{2} M\right)$ are understood as Taylor series in $u . \mathcal{N}[\cdot]$ stands for the normal ordering, which applies only to operators acting at the same site. For them we set

$$
\mathcal{N}\left[\Phi_{\alpha, j}^{\varepsilon} \Psi_{j}^{\varepsilon^{\prime}}\right]=- \begin{cases}\Phi_{\alpha, j}^{\varepsilon} \Psi_{j}^{\varepsilon^{\prime}} & (j>0), \\ -\Psi_{j}^{\varepsilon^{\prime}} \Phi_{\alpha, j}^{\varepsilon} & (j \leq 0)\end{cases}
$$

These operators satisfy a number of remarkable properties. First, the vacuum expectation values can be presented for any local $\mathcal{O}$ as

$$
\begin{gathered}
\frac{\left\langle\operatorname{vac}\left|y^{2 S(0)} \mathcal{O}\right| \operatorname{vac}\right\rangle}{\left\langle\operatorname{vac}\left|y^{2 S(0)}\right| \operatorname{vac}\right\rangle}=\operatorname{tr}^{\alpha}\left(e^{\Omega}(\mathcal{O})\right) \\
\Omega=\frac{i}{\sin \frac{\pi \alpha}{2}} \oint_{\zeta_{1}^{2}=1} \oint_{\zeta_{2}^{2}=1} \omega\left(\zeta_{1} / \zeta_{2}, \alpha\right) \mathbf{b}\left(\zeta_{1}\right) \mathbf{c}\left(\zeta_{2}\right) \mathrm{d} \zeta_{1}^{2} \mathrm{~d} \xi_{2}^{2},
\end{gathered}
$$

where

$$
\omega(\zeta, \alpha)=8 \frac{1+y^{2}}{1-y^{2}} \frac{\xi^{2}}{1-\xi^{4}}\left(1-\xi^{\alpha}\right) .
$$

Let me formulate the rest of properties of these operators in the most general form because formula (2.2) has a direct analogue in the general case.

1. Operators $\mathbf{b}(\zeta), \mathbf{c}(\zeta)$ have the following block structure:

$$
\mathbf{b}(\xi): W_{\alpha, s} \rightarrow W_{\alpha+1, s-1}, \mathbf{c}(\xi): W_{\alpha, s} \rightarrow W_{\alpha-1, s+1} .
$$

Hence operator $\mathbf{b}\left(\xi_{1}\right) \mathbf{c}\left(\xi_{2}\right)$ acts from $W_{\alpha, 0}$ to itself.

2. We have complete anti-commutativity:

$$
\left[\mathbf{b}\left(\zeta_{1}\right) \mathbf{b}\left(\zeta_{2}\right)\right]_{+}=\left[\mathbf{b}\left(\zeta_{1}\right) \mathbf{c}\left(\zeta_{2}\right)\right]_{+}=\left[\mathbf{c}\left(\zeta_{1}\right) \mathbf{c}\left(\zeta_{2}\right)\right]_{+}=0 .
$$

3. We have

$$
\begin{aligned}
& \text { lenght }\left(\mathbf{b}(\zeta)\left(q^{2 \alpha S(0)} \mathcal{O}\right)\right) \leq \text { lenght }\left(q^{2 \alpha S(0)} \mathcal{O}\right) \\
& \text { lenght }\left(\mathbf{b}(\zeta)\left(q^{2 \alpha S(0)} \mathcal{O}\right)\right) \leq \text { lenght }\left(q^{2 \alpha S(0)} \mathcal{O}\right)
\end{aligned}
$$


Moreover, by definition

$\mathbf{b}(\zeta)=\sum_{p=1}^{\infty} \frac{1}{\left(\zeta^{2}-1\right)^{p}} \mathbf{b}_{p} \xi^{-\alpha+s}, \mathbf{c}(\zeta)=\sum_{p=1}^{\infty} \frac{1}{\left(\xi^{2}-1\right)^{p}} \mathbf{c}_{p} \xi^{\alpha-s}$,

then

$$
\begin{aligned}
& \mathbf{b}_{p}\left(q^{2 \alpha S(0)} \mathcal{O}\right)=0, \quad p>\text { lenght }\left(q^{2 \alpha S(0)} \mathcal{O}\right), \\
& \mathbf{c}_{p}\left(q^{2 \alpha S(0)} \mathcal{O}\right)=0, \quad p>\text { lenght }\left(q^{2 \alpha S(0)} \mathcal{O}\right) .
\end{aligned}
$$

This implies that for any $\mathcal{O}$ only a finite number of terms in the series for $e^{\Omega}$ count. Another corollary is that $\mathbf{b}(\zeta)\left(q^{2 \alpha S(k)}\right)=\mathbf{c}(\zeta)\left(q^{2 \alpha S(k)}\right)=0$, so we have translational invariance as was announced.

4. Recall that the local integrals of motion have the form: $I_{p}=\sum d_{j, p}$. So, obviously their adjoint action is well defined on $W_{\alpha}$. Denote $\mathcal{I}_{p}(X)=\left[I_{p}, X\right]$. We have:

$$
\left[\mathcal{I}_{p}, \mathbf{b}(\zeta)\right]=\left[\mathcal{I}_{p}, \mathbf{c}(\zeta)\right]=0 .
$$

This property is very important for self-consistency of our main formula, because the vacuum expectation value of $\mathcal{I}_{p}(X)$ must vanish.

For the moment, all this was for the free fermion case. However, the main result of our research is that one can give an algebraic definition of operators $\mathbf{b}(\zeta), \mathbf{c}(\xi)$ in the case of generic $|q=1|$. They possess the same properties, and the formula for the vacuum expectation values is exactly the same, with the only difference that the function $\omega(\zeta, \alpha)$ should be replaced by:

$$
\begin{aligned}
\omega(\xi, \alpha)= & \frac{4(q \zeta)^{\alpha}}{\left(1+q^{\alpha}\right)^{2}}\left(\frac{q^{-\alpha}}{1-q^{-2} \xi^{2}}-\frac{q^{\alpha}}{1-q^{2} \xi^{2}}\right) \\
& +\int_{-i \infty-0}^{i \infty-0} \zeta^{u+\alpha} \frac{\sin \frac{\pi}{2}(u-v(u+\alpha))}{\sin \frac{\pi}{2} u \cos \frac{\pi v}{2}(u+\alpha)} \mathrm{d} u
\end{aligned}
$$

I shall define the operators $\mathbf{b}(\zeta), \mathbf{c}(\zeta)$ in the last lecture, but I would like to finish the present lecture by completing the set of operators. Namely, we are able to define the creation operators, $\mathbf{b}^{*}(\zeta), \mathbf{s}^{*}(\zeta)$ and an additional operator $\mathbf{t}^{*}(\zeta)$. For the free fermion case one can write explicit formulae for these operators. In the generic case they are constructed similarly to what I shall explain in the next lecture. Their properties are as follows.

1. The block structure of $\mathbf{b}^{*}(\xi), \mathbf{s}^{*}(\zeta), \mathbf{t}^{*}(\zeta)$ is as follows:

$$
\begin{aligned}
& \mathbf{b}^{*}(\zeta): \mathcal{W}_{\alpha-1, s+1} \rightarrow \mathcal{W}_{\alpha, s} \\
& \mathbf{s}^{*}(\xi): \mathcal{W}_{\alpha+1, s-1} \rightarrow \mathcal{W}_{\alpha, s} \\
& \mathbf{t}^{*}(\xi): \mathcal{W}_{\alpha, s} \rightarrow \mathcal{W}_{\alpha, s}
\end{aligned}
$$

2. These operators have the following commutation relations with $\mathbf{b}(\zeta), \mathbf{c}(\zeta)$ :

$$
\begin{aligned}
& {\left[\mathbf{b}\left(\zeta_{1}\right), \mathbf{c}^{*}\left(\zeta_{2}\right)\right]_{+}=\left[\mathbf{c}\left(\zeta_{1}\right), \mathbf{b}^{*}\left(\zeta_{2}\right)\right]_{+} } \\
= & {\left[\mathbf{c}\left(\zeta_{1}\right), \mathbf{t}^{*}\left(\zeta_{2}\right)\right]_{+}=\left[\mathbf{b}\left(\zeta_{1}\right), \mathbf{t}^{*}\left(\zeta_{2}\right)\right]_{+}=0, }
\end{aligned}
$$

$$
\begin{aligned}
& {\left[\mathbf{b}\left(\zeta_{1}\right), \mathbf{b}^{*}\left(\xi_{2}\right)\right]_{+}=\left(\frac{\zeta_{1}}{\zeta_{2}}\right)^{\alpha} \frac{1}{\xi_{1}^{2}-\zeta_{2}^{2}},} \\
& {\left[\mathbf{c}\left(\zeta_{1}\right), \mathbf{c}^{*}\left(\zeta_{2}\right)\right]_{+}=\left(\frac{\zeta_{2}}{\zeta_{1}}\right)^{\alpha} \frac{1}{\zeta_{1}^{2}-\zeta_{2}^{2}} .}
\end{aligned}
$$

3. As functions of $\zeta$ the operators $\mathbf{b}^{*}(\zeta), \mathbf{c}^{*}(\zeta), \mathbf{t}^{*}(\xi)$ are:

$$
\begin{aligned}
& \mathbf{b}^{*}(\xi)=\sum_{p=1}\left(\xi^{2}-1\right)^{p-1} \mathbf{b}_{p}^{*} \xi^{\alpha-s}, \\
& \mathbf{c}^{*}(\xi)=\sum_{p=1}\left(\xi^{2}-1\right)^{p-1} \mathbf{c}_{p}^{*} \xi^{\alpha-s}, \\
& \mathbf{t}^{*}(\xi)=\sum_{p=1}\left(\xi^{2}-1\right)^{p-1} \mathbf{t}_{p}^{*}
\end{aligned}
$$

4. The operators $\mathbf{b}^{*}(\xi), \mathbf{c}^{*}(\zeta), \mathbf{t}^{*}(\zeta)$ acting on $q^{2 \alpha S(k)}$ create the space $W_{\alpha}$. The locality is respected due to the properties:

$$
\begin{aligned}
& \text { lenght }\left(\mathbf{b}_{p}^{*}\left(q^{2 \alpha S(0)} \mathcal{O}\right)\right) \leq \text { lenght }\left(q^{2 \alpha S(0)} \mathcal{O}\right)+p, \\
& \text { lenght }\left(\mathbf{c}_{p}^{*}\left(q^{2 \alpha S(0)} \mathcal{O}\right)\right) \leq \text { lenght }\left(q^{2 \alpha S(0)} \mathcal{O}\right)+p, \\
& \text { lenght }\left(\mathbf{t}_{p}^{*}\left(q^{2 \alpha S(0)} \mathcal{O}\right)\right) \leq \text { lenght }\left(q^{2 \alpha S(0)} \mathcal{O}\right)+p .
\end{aligned}
$$

5. The operators $\mathbf{b}^{*}(\zeta), \mathbf{c}^{*}(\zeta), \mathbf{t}^{*}(\zeta)$ respect $\mathbf{t r}^{\alpha}$ :

$$
\begin{aligned}
& \operatorname{tr}^{\alpha}\left(\mathbf{b}^{*}(\xi)(X)\right)=\operatorname{tr}^{\alpha}\left(\mathbf{c}^{*}(\zeta)(X)\right)=0, \\
& \operatorname{tr}^{\alpha}\left(\mathbf{t}^{*}(\zeta)(X)\right)=\operatorname{tr}^{\alpha}(X) .
\end{aligned}
$$

For the vacuum expectation value we have:

$$
\begin{aligned}
& \frac{\left\langle\operatorname{vac}\left|\mathbf{b}^{*}\left(\zeta_{1}^{-}\right) \cdots \mathbf{b}^{*}\left(\zeta_{p}^{-}\right) \mathbf{c}^{*}\left(\zeta_{1}^{+}\right) \cdots \mathbf{c}^{*}\left(\zeta_{p}^{+}\right) \mathbf{t}^{*}\left(\zeta_{1}^{0}\right) \cdots \mathbf{t}^{*}\left(\zeta_{q}^{0}\right) q^{2 \alpha S(0)}\right| \operatorname{vac}\right\rangle}{\left\langle\operatorname{vac}\left|q^{2 \alpha S(0)}\right| \operatorname{vac}\right\rangle}= \\
& \quad=\operatorname{det} w\left(\zeta_{j}^{+} / \zeta_{j}^{-}, \alpha\right)
\end{aligned}
$$

I think this is a good point to finish this lecture.

\section{Lecture 3.}

In the previous lecture I claimed that the space of operators $W_{\alpha}$ can be organized in such a way that the vacuum expectation values are easy to calculate. Namely, I claimed that there are operators $\mathbf{b}(\zeta), \mathbf{c}(\zeta), \mathbf{b}^{*}(\zeta), \mathbf{c}^{*}(\xi), \mathbf{t}^{*}(\zeta)$ which can be constructed algebraically, and which provide this organization of $W_{\alpha}$. But for the moment I described some of these operators for the case of free ferminos only. Now I shall explain the general construction, but I think I shall not be able to do this for all the operators. I shall therefore restrict myself to the operator $\mathbf{c}(\zeta)$. If the construction of these operators is clear to you at the end of this lecture I shall be quite happy. Other operators are constructed using similar means.

First, let me prepare our notation for the $L$-operators. Consider the quantum affine algebra $U_{q}\left(\widehat{\left(\mathfrak{s}_{2}\right)}\right)$. The universal $R$-matrix of this algebra belongs to the tensor product $\mathfrak{b}_{+} \otimes \mathfrak{b}_{-}$ of its two Borel subalgebras. By an $L$-operator we mean its image under an algebra map $\mathfrak{b}_{+} \otimes \mathfrak{b}_{-} \rightarrow N_{1} \otimes N_{2}$, where $N_{1}$, $N_{2}$ are some algebras. I shall always take $N_{2}$ to be the algebra 
$M=\operatorname{Mat}(2, \mathbb{C})$ of $2 \times 2$ matrices. As for $N_{1}$ I make several choices: $U_{q}\left(\mathfrak{s}_{2}\right), M$, the $q$-oscillator algebra Osc (see below) or Osc $\otimes M^{ \pm}$, where $M^{ \pm} \subset M$ are the subalgebras of upper and lower triangular matrices. For economy of symbols, I use the same letter $L$ to designate these various $L$-operators. I shall put indices, indicating to which tensor product of the algebras they belong. We use $j, k, \ldots$ as labels for the lattice sites, and $a$, $b, \ldots$ as labels for the 'auxiliary' two-dimensional space. Accordingly I write the matrix algebra as $M_{j}$ or $M_{a}$. Capital letters $A, B, \ldots$ will indicate the $q$-oscillator algebra Osc. Finally, for $O s c \otimes M^{ \pm}$I use pairs of indices such as $\{A, a\}$.

We already had the $L$-operator $L_{a, j}(\zeta)$, which is essentially the image of the universal $R$-matrix under the map of both $\mathfrak{b}_{+}, \mathfrak{b}_{-}$to $\operatorname{Mat}(\mathbb{C}, 2)$.

The next case is due originally to Bazhanov, Lukyanov and Zamolodchikov [9, 10, 11]. Let us consider the $q$-oscillators a, a ${ }^{*}$ satisfying

$$
\mathbf{a} \mathbf{a}^{*}-q^{2} \mathbf{a}^{*} \mathbf{a}=1-q^{2} .
$$

It is convenient to introduce one more element $q^{D}$ such that

$$
\begin{aligned}
& q^{D} \mathbf{a}^{*}=\mathbf{a}^{*} q^{D+1}, \quad q^{D} a=a q^{D-1}, \\
& \mathbf{a}^{*} \mathbf{a}=1-q^{2 D}, \quad \mathbf{a} \mathbf{a}^{*}=1-q^{2 D+2} .
\end{aligned}
$$

Denote by $O s c$ the algebra generated by a, $\mathbf{a}^{*}, q^{ \pm D}$ with the above relations. We consider the following two representations of $O s c$,

$$
W^{+}=\bigoplus_{k=0}^{\infty} C|k\rangle, \quad \mathbf{a}^{*}|k-1\rangle=|k\rangle, \quad D|k\rangle=k|k\rangle, \quad \mathbf{a}|0\rangle=0
$$

$$
W^{-}=\bigoplus_{k=-\infty}^{-1} C|k\rangle, \quad \mathbf{a}^{*}|k+1\rangle=|k\rangle, \quad D|k\rangle=k|k\rangle, \quad \mathbf{a}^{*}|-1\rangle=0
$$

In the root of the unity case, if $r$ is the smallest positive integer such that $q^{2 r}=1$, we consider the $r$-dimensional quotient of $W^{ \pm}$generated by $|0\rangle$ or $|-1\rangle$.

My goal is not to give a complete description of the construction, but rather to consider an example. So, I shall explain how to construct the operator $\mathbf{c}(\zeta)$. This operator has the block structure explained before. Let me denote by $\mathbf{c}(\xi)$ its block acting from $W_{\alpha}$ to $W_{\alpha-1}$.

Returning to the $L$-operators, we introduce the following one, which is the image of the universal $R$-matrix for $N_{1}=O s c$, $N_{2}=\operatorname{Mat}(2, \mathbb{C})$ :

$$
\begin{aligned}
& L_{A, j}(\zeta)=i \zeta^{-\frac{1}{2}} q^{-\frac{1}{4}}\left(\begin{array}{cc}
1-\xi^{2} q^{2 D_{A}+2} & -\zeta \mathbf{a}_{A} \\
-\zeta \mathbf{a}_{A}^{*} & 1
\end{array}\right)_{j} \\
& \left(\begin{array}{cc}
q^{-D_{A}} & 0 \\
0 & q^{D_{A}}
\end{array}\right)_{j} \in O s c_{A} \otimes M_{j} .
\end{aligned}
$$

This $L$-operator satisfies the crossing symmetry relation:

$$
L_{A, j}(\zeta)^{-1}=\frac{1}{\zeta-\zeta^{-1}} \bar{L}_{A, j}(\zeta),
$$

where we have set

$$
\bar{L}_{A, j}(\xi)=\sigma_{j}^{2} L_{A, j}\left(\zeta q^{-1}\right)^{t_{j}} \sigma_{j}^{2},
$$

and $t_{j}$ stands for the transposition in $M_{j}$.

Consider the product $L_{a, j}(\xi) L_{A, j}(\zeta)$. It is well known that this product can be brought to a triangular form, giving rise in particular to Baxter's 'TQ-equation' for transfer matrices. Introduce $F_{a, A}=1-\mathbf{a}_{A} \sigma_{a}^{+}$, then we have the fusion relation:

$$
\begin{aligned}
L_{\{a, A\}, j}(\xi) & =F_{a, A}^{-1} L_{a, j}(\zeta) L_{A, j}(\zeta) F_{a, A} \\
& =\left(\begin{array}{cc}
\zeta-\xi^{-1} & 0 \\
\left(q-q^{-1}\right) \sigma_{j}^{+} & \zeta q-\zeta^{-1} q^{-1}
\end{array}\right)_{a} \\
& \left(\begin{array}{cc}
L_{A}(\zeta q) q^{-\sigma_{j}^{3} / 2} & 0 \\
0 & L_{A}(\zeta q) q^{-\sigma_{j}^{3} / 2}
\end{array}\right)_{a} .
\end{aligned}
$$

We shall also need

$$
\begin{aligned}
L_{\{a, A\}, j}(\zeta)^{-1} & =\frac{1}{\left(\zeta-\xi^{-1}\right)\left(\zeta q-\zeta^{-1} q^{-1}\right)\left(\zeta q-\zeta^{-1} q\right)} \\
& \times\left(\begin{array}{cc}
q^{\sigma_{j}^{3} / 2} \bar{L}_{A}(\zeta q) & 0 \\
0 & q^{-\sigma_{j}^{3} / 2} \bar{L}_{A}\left(\zeta q^{-1}\right)
\end{array}\right)_{a} \\
& \left(\begin{array}{cc}
\zeta q^{-1}-\xi^{-1} q & 0 \\
-\left(q-q^{-1}\right) \sigma_{j}^{+} & \zeta-\xi^{-1}
\end{array}\right)_{a} .
\end{aligned}
$$

Let us consider the finite chain with sites from $k$ to $l$. By $M_{[k, l]}$ I shall denote $\operatorname{Mat}(2, \mathbb{C})^{\otimes(l-k+1)}$. As usual, the main object is the monodromy matrix:

$$
T_{x,[k, l]}^{ \pm}(\zeta)=L_{x, l}^{ \pm}(\zeta) \cdots L_{x, k}^{ \pm}(\zeta),
$$

where $x$ stands for any auxiliary algebra. However, contrary to the usual situation I want to act on the operators, so, I introduce the adjoint action:

$$
\mathcal{T}_{x,[k, l]}(\zeta)(\cdot)=T_{x,[k, l]}^{ \pm}(\zeta) \cdot T_{x,[k, l]}^{ \pm}(\zeta)^{-1} .
$$

Consider $X \in M_{k, l}$ and define

$$
\begin{aligned}
\left(\begin{array}{cc}
\mathbb{A}_{A,[k, l]}(\xi, \alpha) & 0 \\
\mathbb{C}_{A,[k, l]}(\xi, \alpha) & \mathbb{D}_{A,[k, l]}(\zeta, \alpha)
\end{array}\right)_{a}(X)= & \mathbb{T}_{\{a, A\},[k, l]}(\zeta) \\
& \left(q^{(\alpha-1)\left(2 D_{A}+\sigma_{a}^{3}\right)} q^{\left.-2 S_{[k, l]} X\right)},\right.
\end{aligned}
$$

where $S_{[k, l]}=\frac{1}{2} \sum_{j=k}^{j} \sigma_{j}^{3}$. From the fusion relation we obtain:

$$
\begin{aligned}
& \mathbb{A}_{A,[k, l]}(\zeta, \alpha)(X)=q^{\alpha-1} \mathbb{T}_{A,[k, l]}(\zeta, q) q^{-\mathbb{S}}\left(q^{2(\alpha-1) D_{A}} q^{\left.-2 S_{[k, l]} X\right),}\right. \\
& \mathbb{D}_{A,[k, l]}(\xi, \alpha)(X)=q^{-\alpha+1} \mathbb{T}_{A,[k, l]}\left(\xi, q^{-1}\right) q^{-\mathbb{S}}\left(q^{2(\alpha-1) D_{A}} q^{\left.-2 S_{[k, l]} X\right),}\right.
\end{aligned}
$$

where $\mathbb{S}$ stands for the adjoint action of total spin.

Now I introduce the most important object:

$$
\mathbf{c}_{[k, l]}^{(0)}(\xi, \alpha)(X)=\operatorname{Tr}_{A}^{+}\left(\mathbb{C}_{A,[k, l]}(\xi, \alpha) \xi^{\alpha-S}(X),\right.
$$


the trace is taken with respect to $W^{+}$, it converges for $\left|q^{\alpha}\right|<1$, and continues analytically to the other $\alpha$.

There is one obvious property of $\mathbf{c}_{[k, l]}^{(0)}(\zeta, \alpha)$ :

$$
\mathbf{c}_{[k, l]}^{(0)}(\xi, \alpha)\left(q^{\alpha \sigma_{k}^{3}} X_{[k+1, l]}\right)=q^{(\alpha-1) \sigma_{k}^{3}} \mathbf{c}_{[k, l]}^{(0)}(\xi, \alpha)\left(X_{[k+1, l]}\right) .
$$

It follows from the definition of the adjoint and $U(1)$-symmetry of the $L$-operator. We call this property the first reduction relation.

In addition there is another property which is a result of non-trivial calculation:

$\mathbf{c}_{[k, l]}^{(0)}(\zeta, \alpha)\left(X_{[k, l-1]} I_{l}\right)=\mathbf{c}_{[k, l-1]}^{(0)}(\zeta, \alpha)\left(X_{[k, l-1]}\right) I_{l}+\Delta(F(\zeta))$,

where $\Delta(f(\xi))=f(\xi q)-f\left(\xi q^{-1}\right)$, the explicit expression for $F(\zeta)$ is irrelevant here. So, the reduction relation from the right is satisfied up to the " $q$-exact form". In classical mathematics the additional term is usually eliminated by integrating over the closed cycle. Here it is similar. Obviously, $\mathbf{c}_{[k, l]}^{(0)}(\zeta, \alpha)$ is singular at $\xi^{2}=1, q^{2}, q^{-2}$. Then the above relation implies that for

$$
\mathbf{c}_{[k, l]}(\zeta, a)=\operatorname{sing}_{\zeta^{2}=1}\left(\mathbf{c}_{[k, l]}^{(0)}(\zeta q, \alpha)+\mathbf{c}_{[k, l]}^{(0)}\left(\zeta q^{-1}, \alpha\right)\right)
$$

we have

$$
\mathbf{c}_{[k, l]}(\zeta, a)\left(X_{[k, l-1]} I_{l}\right)=\mathbf{c}_{[k, l-1]}(\zeta, a)\left(X_{[k, l-1]}\right) I_{l} .
$$

This is the second reduction relation. Now we are able to define the operator $\mathbf{c}(\xi, a)$ acting from $W_{\alpha}$ to $W_{\alpha-1}$ taking the inductive limit. Indeed consider $X \in W_{\alpha}$, denote by $X_{[k, l]}$ its restriction to the interval $[k, l]$. Then

$$
\mathbf{c}(\xi, a)(X)=\lim _{k \rightarrow \infty, l \rightarrow \infty} \mathbf{c}_{[k, l]}(\zeta, a)\left(X_{[k, l]}\right)
$$

due to the reduction relation the limit is well-defined since for a large enough interval $[k, l]$ the sequence stabilizes!

The commutation relations are proved similarly. I do not go into details, but using the $R$-matrix one can show that

$$
\begin{aligned}
\mathbf{c}_{[k, l]}^{(0)}\left(\zeta_{1}, \alpha-1\right) \mathbf{c}_{[k, l]}^{(0)}\left(\zeta_{2}, \alpha\right) & +\mathbf{c}_{[k, l]}^{(0)}\left(\zeta_{2}, \alpha-1\right) \mathbf{c}_{[k, l]}^{(0)}\left(\zeta_{1}, \alpha\right) \\
& =\Delta_{\zeta_{1}}\left(F\left(\zeta_{1}, \zeta_{2}\right)\right)+\Delta_{\zeta_{2}}\left(F\left(\zeta_{2}, \zeta_{1}\right)\right),
\end{aligned}
$$

so, there is anticommutativity up to the "q-exact 2-form". Again, "integrating over the closed cycle" (passing to $\mathbf{c}(\zeta)$ ) one obtains anticommutativity.

Let me finish these lecture with some general remarks. The construction of other operators is not very different from c, but for $\mathbf{b}^{*}$ and $\mathbf{c}^{*}$ some additional work has to be done. The commutation relations are not always easy to prove because the $R$-matrices are not always applicable.

I did not say anything about the derivation of our formula for the vacuum expectation values for generic $q$. Actually, it was obtained as a result of a long transformation of the integral formula by Jimbo and Miwa [12]. However, I find quite unsatisfactory that we have such a complicated derivation of such a simple result. I hope to find a more direct proof.

\section{References}

[1] Boos, H., Jimbo, M., Miwa, T., Smirnov, F., Takeyama, Y.: A Recursion Formula for the Correlation Functions of an Inhomogeneous XXX Model. Algebra and Analysis, (2005), p. 115-159.

[2] Boos, H., Jimbo, M., Miwa, T., Smirnov, F., Takeyama, Y.: Reduced qKZ Equation and Correlation Functions of the XXZ Model. Commun. Math. Phys. (2006), p. 245-276.

[3] Boos, H., Jimbo, M., Miwa, T., Smirnov, F., Takeyama, Y.: Traces on the Sklyanin Algebra and Correlation Functions of the Eight-Vertex Model.J. Phys. A: Math. Gen. (2005), p. 7629-7659.

[4] Boos, H., Jimbo, M., Miwa, T., Smirnov, F., Takeyama, Y.: Density Matrix of a Finite Sub-Chain of the Heisenberg Anti-Ferromagnet, Lett. Math. Phys. (2006), p. 201-208.

[5] Boos, H., Jimbo, M., Miwa, T., Smirnov, F., Takeyama, Y.: Algebraic Representation of Correlation Functions in Integrable Spin Chains. Ann. Henri Poincaré (2006), p. 1395-1428.

[6] Boos, H., Jimbo, M., Miwa, T., Smirnov, F., Takeyama, Y.: Hidden Grassmann Structure in the XXZ Model. Commun. Math. Phys., (2007), p. 263-281.

[7] Boos, H., Jimbo, M., Miwa, T., Smirnov, F., Takeyama, Y.: Fermionic Basis for Space of Operators in the XXZ Model. hep-th/0702086

[8] Boos, H., Jimbo, M., Miwa, T., Smirnov, F., Takeyama, Y.: Hidden Grassmann Structure in the XXZ Model II. Creation Operators. In preparation.

[9] Bazhanov, V., Lukyanov, S., Zamolodchikov, A.: Integrable Structure of Conformal Field Theory, Quantum KdV Theory and Thermodynamic Bethe Ansatz, Commun. Math. Phys., (1996), p. 381-398.

[10] Bazhanov, V., Lukyanov, S., Zamolodchikov, A.: Integrable Structure of Conformal Field theory II. Q-Operator and DDV Equation, Commun. Math. Phys. (1997), p. 247-278.

[11] Bazhanov, V., Lukyanov, S., Zamolodchikov, A.: Integrable Structure of Conformal Field Theory III. The Yang-Baxter Relation, Commun. Math. Phys. (1999), p. 297-324.

[12] Jimbo, M., Miwa, T.: Algebraic Analysis of Solvable Lattice Models, Reg. Conf. Ser. in Math., AMS,. Vol. 85 (1995).

\section{Fedor Smirnov}

e-mail: smirnov@lpthe.jussieu.fr

FS (membre du CNRS):

Laboratoire de Physique Théorique et Hautes Energies, Université Pierre et Marie Curie, Tour 161 étage

4 Place Jussieu

75252 Paris Cedex 05, France 